MS43-02

\section{Computational prediction of MOF structures from the entire periodic table: AuToGraFS + UFF4MOF}

Matthew Addicoat ${ }^{1}$

1. School of Science and Technology, Nottingham, United Kingdom email: matthew.addicoat@ntu.ac.uk

MOFs are inorganic-organic composite materials built by combining individually stable building blocks. Dozens of inorganic connectors may be linked via a nearly infinite variety of organic molecules in hundreds of topologies, resulting in a plethora of possible structures. The accurate and low-cost prediction of these structures is important for guiding synthetic efforts toward applications.

To meet this need of providing high quality structures of arbitrary frameworks, we developed AuToGraFS: the Automatic Topological Generator for Framework Structures [1]. AuToGraFS is open-source software, which employs a database of SBUs to create framework structure models using topologies sourced from the Reticular Chemistry Structure Resource (RCSR).

An integral part of AuToGraFS is a MOF-specific extension to Rappé's Universal Force Field, UFF4MOF [2]. The UFF$4 \mathrm{MOF}$ extension to UFF contains parameters for all transition metal environments found in the Computation-Ready Experimental (CoRE) Database and specific parameters for paddlewheels, $\mathrm{Zn}_{4} \mathrm{O}$ and $\mathrm{M}_{3} \mathrm{O}$ building blocks. Hydrogen-bonded frameworks are also possible [3]. With these two tools, we produce highly accurate structures with less than $2 \%$ error on cell parameters, even on cells as large as 20,000 atoms.

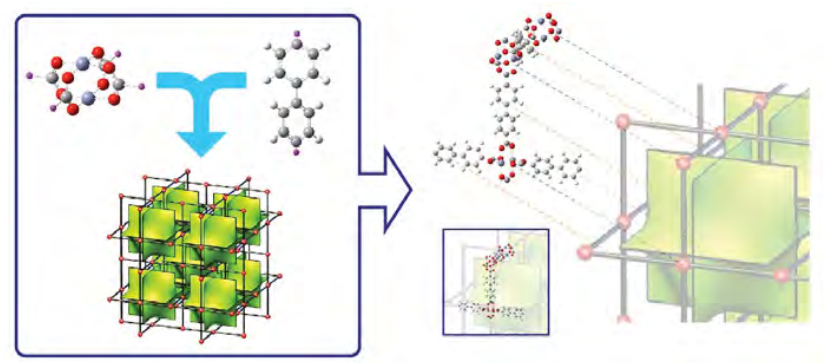

References:

[1]. Addicoat et al. J Phys Chem A, 118, 9607-9614 (2014), https:// github.com/maddicoat/AuToGraFS

[2]. Addicoat et al. J Chem Theory Comput, 10, 880-891 (2014), J

Chem Theory Comput, 12, 5215-5225 (2016)

[3]. Coupry, D. E. et al. J Chem Phys, 147, 161705, (2017)

Keywords: Metal-organic frameworks, Covalent organic frameworks, AuToGraFS

\section{MS43-03}

\section{Complex approach to analysis of crystal structures based on unified topological model}

Eugeny Alexandrov ${ }^{1}$, Andrey Golov ${ }^{1}$, Alexander Shevchenko ${ }^{1}$

1. SCTMS at Samara University and Samara State Technical University, Samara, Russia

email: aleksandrov_ev1@mail.ru

We present a unified topological model for analysis of connectivity in crystal structures. In this model, we represent crystal structure as a periodic simple graph (net), whose vertices and edges have particular weights. The resulting weighted net combines connectivity of chemical species with their descriptors. This approach provides heuristic analysis of crystal structures, modeling crystal growth and physical properties of substances (e.g. ionic conductivity), and finding structural relations at different levels of crystal organization.

The descriptors characterize the overall net (e.g. topological type), structural groups (e.g. coordination symbol), interatomic contacts (e.g. length), and voids (e.g. radii). We also propose two groups of new descriptors for interatomic contacts and voids. The former group includes bond pathways and parameters of electron density at the bond critical point: the electron density $\rho\left(r_{c p}\right), \Delta \rho\left(r_{c p}\right)$, density of potential and kinetic energy. The latter group contains area and volume of van der Waals spheres of atoms and channel systems $(\mathrm{ChS})$, periodicity of $\mathrm{ChS}, h k l$ of $\mathrm{ChS}$, porosity, radius of included and free spheres.

The following new algorithms are developed to use the descriptors within the model:

1) scanning of Voronoi net (a net of vertices and edges of Voronoi polyhedra) for analysis of the channels and cavities in a crystal structure;

2) approximation of the elementary void by Voronoi polyhedron whose faces are shifted to the van-der-Waals surface;

3) searching for the accessible channels system;

4) splitting the structure net to subgraphs (ligands, clusters, homoleptic groups) and their topological analysis.

All the algorithms are implemented in the ToposPro package (http://topospro.com/).

Using the algorithms two new topological databases were created to be used in the screening and design of MOF structures: the database of structural building units (more than 5000 entries) implemented in the TORIS client (http:// topospro.com/software/toris/) and the database of cavities and channels in more than 14000 MOFs.

New algorithms and descriptors were successfully used for assessment of mechanical stability and adsorption properties of some breathing and rigid MOFs, checking for isomorphism of 502 MOF structures from the CoRe MOF database [1], and searching for structural relations of 11 new coordination polymers $[2,3]$. We discuss the applicability of the topological model for the development of crystallographic knowledge databases.

The authors thank the Russian Science Foundation (Grant No. 16-13-10158), the Ministry of Education and Science of the Russian Federation (Grant No. 1.6101.2017/9.10), and the Russian Foundation for Basic Research (Grant No. 17-57-10001). 